\title{
Letter to the editor: Estimation of milk fatty acid yield: A comment on Glasser et al. (2007)
}

\author{
J. A. Stamey, ${ }^{*}$ B. A. Corl, ${ }^{* 1}$ P. Y. Chouinard, $†$ and J. K. Drackley $\ddagger$ \\ ${ }^{*}$ Department of Dairy Science, Virginia Polytechnic Institute and State University, Blacksburg 24061 \\ †Département des Sciences Animales, Université Laval, Québec, QC, Canada G1V 0A6 \\ ‡Department of Animal Sciences, University of Illinois, Urbana 61801
}

Triglyceride, the major lipid of milk fat, is composed of 3 fatty acids (FA) esterified to glycerol. For nutritional studies of milk fat quality and measurement of fatty acid transfer to milk fat, quantifying secreted FA in milk fat is important. A single oxygen within the ester bond links each FA to the glycerol backbone. Each ester bond for TG synthesis requires the transfer of fatty acyl-coenzyme A (CoA) to the glycerol backbone (Pynadath and Kumar, 1964; Kinsella and Gross, 1973), a mechanism highlighted by the requirement for $\mathrm{CoA}$ for the esterification of palmitate (Askew et al., 1971).

Glasser et al. (2007) proposed a method to estimate milk FA yield by using a constant coefficient of $93.3 \%$ to represent the proportion of FA in total milk lipids. They correctly point out that fatty acid yields reported in most publications are yields of fatty acyl groups, radicals without the hydroxyl $(-\mathrm{OH})$ of the carboxyl group. Glasser et al. (2007) proposed this coefficient to more easily estimate balances of nutritional value and NEFA flow by calculating FA yields rather than fatty acyl yields.

The source of the oxygen molecule within the ester bond must be taken into account in the calculation of FA and glycerol yields in milk fat. Glasser et al. (2007) attribute the oxygen of the ester bond to the $-\mathrm{OH}$ group of the FA, including only the mass of the carbon backbone of glycerol in calculating its yield, which is mechanistically inaccurate. As FA-CoA is the substrate for esterification, oxygen within the ester bond must originate from the $-\mathrm{OH}$ group of glycerol. The $-\mathrm{OH}$ groups of glycerol account for almost 50\% of glycerol's molecular weight. In the calculation of yields of glycerol and individual milk FA, therefore, Glasser et al. (2007) underestimate the contribution of glycerol to milk lipid mass. By calculating FA yield rather than fatty acyl

Received April 30, 2010.

Accepted May 13, 2010.

${ }^{1}$ Corresponding author: bcorl@vt.edu yield, the method of Glasser et al. (2007) does not represent mammary gland mechanisms of esterification.

Glasser et al. (2007) acknowledge that this is mechanistically inaccurate, but feel that calculating fatty acid yield will standardize reporting across publications and allow readers to better calculate fatty acid flows to the mammary gland. We advocate for calculation of fatty acyl yield because it is mechanistically accurate, representing mammary epithelial cell esterification. Experimental treatments that change the proportions of short and long chain fatty acids in milk fat alter the amount of glycerol present in milk fat as discussed by Schauff and Clark (1992). Furthermore, we do not agree with Glasser et al. (2007) that calculation of fatty acid yield is required for readers to understand fatty acid flows. To be accurate, yields in publications should clearly state that fatty acyl yield is presented rather than fatty acid yield, allowing the reader to apply conversion factors to fatty acyl mass should fatty acid mass be of interest.

In conclusion, as the coefficient proposed by Glasser et al. (2007) does not accurately portray the esterification of fatty acids within the mammary gland and because it is a simple calculation to convert fatty acyl yield to fatty acid yield, we feel that fatty acyl yield should be reported and clearly indicated as such in publications.

\section{REFERENCES}

Askew, E. W., R. S. Emery, and J. W. Thomas. 1971. Characteristics of fatty acid esterification by homogenates of bovine mammary tissue. Lipids 6:326-331.

Glasser, F., M. Doreau, A. Ferlay, and Y. Chilliard. 2007. Technical note: Estimation of milk fatty acid yield from milk fat data. J. Dairy Sci. 90:2302-2304.

Kinsella, J. E., and M. Gross. 1973. Palmitic acid and initiation of mammary glyceride synthesis via phosphatidic acid. Biochim. Biophys. Acta 316:109-113.

Pynadath, T. I., and S. Kumar. 1964. Incorporation of short- and long-chain fatty acids into glycerides by lactating-goat mammary tissue. Biochim. Biophys. Acta 84:251-263.

Schauff, D. J., and J. H. Clark. 1992. Effects of feeding diets containing calcium salts of long-chain fatty acids to lactating dairy cows. J. Dairy Sci. 75:2990-3002. 